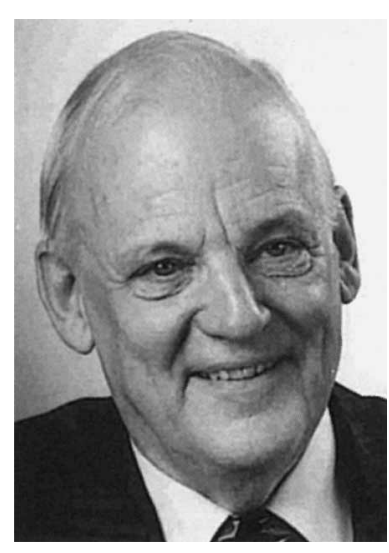

Gösta Samuelson gosta.samuelson@htu.se

\title{
Salt iodization: effectively combating iodine deficiency
}

I odine deficiency is a major nutritional problem worldwide. About 2.2 billion people are at risk of deficiency, according to the World Health Organization (WHO) (Hetzel BS. Bull World Health Organ 2002;80:410-17). In infants, a low blood level of thyroid hormones is the main factor responsible for damaging the developing brain. In Scandinavia, as in many other countries, a neonatal thyroid-stimulating hormone (TSH) screening programme has been successful in the early detection of congenital hypothyroidism. However, in both developed and developing countries a low intake of dietary iodine results in iodine deficiency. The most effective approach is to prevent iodine deficiency through universal salt iodization. WHO is now performing a worldwide mapping of iodine deficiency disorders (WHO Global IDD Database, www3.who.int/whosis/micronutrient/). In this issue of Scandinavian Journal of Nutrition (SJN), Maria Andersson et al., from WHO, describe the ongoing work. Furthermore, a study of iodine intake and iodine status in two groups of Norwegians is published by Lisbeth Dahl et al.

In Norway the main sources of dietary iodine are milk and dairy products, as well as fish and fish products, which contribute about $80 \%$ of the iodine intake. In other European countries iodinated salt, meat, bread and milk products contribute a major part of the iodine intake. In the Norwegian study the contribution of iodine from supplements or from iodized salt was not included in the calculations, since only some brands of table salt in Norway are fortified with iodine. The study by Dahl et al. combines an estimation of iodine intake in the diet with urinary iodine concentrations and serum levels of TSH and free thyroid hormone. Some population groups have a very low intake of iodine, which is an important finding. The material is, however, relatively small and the methodology is debatable. Nationwide studies in all countries are needed, and in Norway such a study is in progress. The authors conclude that iodization of table salt in Norway is too low to secure sufficient iodine intake for all people.

\section{Diets for health without scientific evidence}

A lot of books and websites are now overloading the market with all kinds of dietary advice regarding better health and weight loss. Methods according to M Montignac, F Lindberg, R Atkins and F Paulún are some examples.

The prevalence of overweight and obesity in children, adolescents and adults of all ages is probably the cause of the great interest in finding easy methods to decrease weight and improve health. In an invited commentary by Jarl Torgerson in this issue of $\mathrm{SJN}$, the low-carbohydrate dieting method is commented on, based on a recently published systematic review article on this topic by Bravata et al. in JAMA (2003;348: 2074-81). Torgerson concludes that "there is not enough information to make clear recommenda- tions for or against that type of diet in obesity treatment".

To prevent gains in weight and obesity, reduction in dietary energy intake and increased physical activity are of great significance. Therefore, "big meals" with a lot of energy from all kinds of energyyielding foods as fat-rich products and sugar-rich beverages should be avoided or reduced, as should frequent intake of soft drinks and alcohol. The best advice for good health is still to eat a varied diet high in carbohydrates with a lot of fruits and vegetables in combination with fish, lean meat, and unsaturated fats. This also means a consequent meal pattern without stress, and regular physical activity. These old pieces of advice gain increasing scientific support, and are of vital importance for everybody's health. 